\title{
Quick regression of brain metastases from lung adenocarcinoma with epidermal growth factor receptor-tyrosine kinase inhibitor treatment: A case report and literature review
}

\author{
HUAQIONG HUANG* ${ }^{*}$ SHUANGLI ZHU*, SHAOBIN WANG and WEN LI \\ Department of Respiratory Medicine, Second Affiliated Hospital of Zhejiang University \\ School of Medicine, Hangzhou, Zhejiang 310009, P.R. China
}

Received December 30, 2015; Accepted March 16, 2016

DOI: $10.3892 / \operatorname{mco} .2016 .916$

\begin{abstract}
Brain metastasis (BM) commonly occurs in patients with advanced lung cancer, and is associated with poor prognosis and limited treatment options, particularly for end-stage patients who are in poor physical and mental state. We herein present a case of lung adenocarcinoma with BM, as revealed by tumor marker and imaging studies. The patient was a 74-year-old woman who was diagnosed with lung adenocarcinoma with several metastatic lesions in the mediastinal lymph nodes, bone and brain. The patient underwent two cycles of chemotherapy, but the cancer recurred with enlarged $\mathrm{BM}$, resulting in confusion and body dysfunction. The patient then received epidermal growth factor receptor-tyrosine kinase inhibitor (EGFR-TKI) therapy with icotinib. After approximately $12 \mathrm{~h}$ of treatment the symptoms disappeared, and the metastatic lesions in the brain largely regressed in the following months. Our case indicates that the EGFR-TKI icotinib may provide a rapid and safe approach for emergency situations with BM from lung adenocarcinoma.
\end{abstract}

\section{Introduction}

Lung cancer is the leading cause of cancer-related mortality worldwide (1). Brain metastasis (BM) is a common secondary localization of the disease in lung cancer patients, encountered in $\sim 7.4 \%$ of non-small-cell lung cancer (NSCLC) cases at diagnosis (2), and $25-30 \%$ of the cases over the course of the disease (3). The prognosis of NSCLC with BM is poor

Correspondence to: Dr Wen Li, Department of Respiratory Medicine, Second Affiliated Hospital of Zhejiang University School of Medicine, 88 Jiefang Road, Hangzhou, Zhejiang 310009, P.R. China

E-mail: liwenzjhz0408@163.com

${ }^{*}$ Contributed equally

Key words: lung, adenocarcinoma, brain metastasis, epidermal growth factor receptor-tyrosine kinase inhibitor and the mortality is high $(4,5)$. The most common treatment for these patients is radiation therapy $(6,7)$; however, the therapeutic options are limited in an emergency setting, as well as for end-stage patients. In this report, we present the case of a NSCLC patient with BM who received epidermal growth factor receptor-tyrosine kinase inhibitor (EGFR-TKI) therapy, with quick regression of the symptoms. The present case suggests that EGFR-TKI therapy may be effective for late-stage NSCLC patients, or in an emergency setting.

\section{Case report}

On, November 19, 2013, a 74-year-old female patient was admitted to the Respiratory Department of The Second Affiliated Hospital of Zhejiang University School of Medicine (Hangzhou, China) complaining of persistent cough and progressive dyspnea. The patient had already undergone a lung computed tomography (CT) scan at a local hospital, which revealed a mass in the lower lobe of the left lung, combined with left pleural effusion and mediastinal lymph node enlargement. Following admission, a contrast-enhanced CT revealed a mass sized $\sim 98 \times 79 \mathrm{~mm}$, blocking the left main bronchus and invading the left pulmonary artery and its branches. Bronchoscopy was performed and a tumor was identified in the left lower airway. Pathological examination of a biopsy specimen identified the lesion as lung adenocarcinoma. Genetic analysis identified an exon 19 EGFR mutation in the patient. Following magnetic resonance imaging and ultrasound studies, metastases were found in the brain, skull, adrenal gland and abdominal lymph nodes. No significant symptoms or body dysfunction were associated with the metastases, except for a painless horn-like protrusion on the right side of the forehead. Administration of EGFR-TKI therapy was immediately recommended. However, the patient's family rejected this treatment strategy due to its high cost, and opted for chemotherapy instead. Pemetrexed disodium $\left(75 \mathrm{mg} / \mathrm{m}^{2}\right)$ and carboplatin (area under the curve $=5$ ) were administered every 21 days for a total of two cycles.

Eleven days after the second cycle of chemotherapy, the patient was admitted to our hospital due to left body dysfunction for 5 days. The physical examination revealed no changes in muscle tone. Muscle strength was rated 0/5 in the left upper 


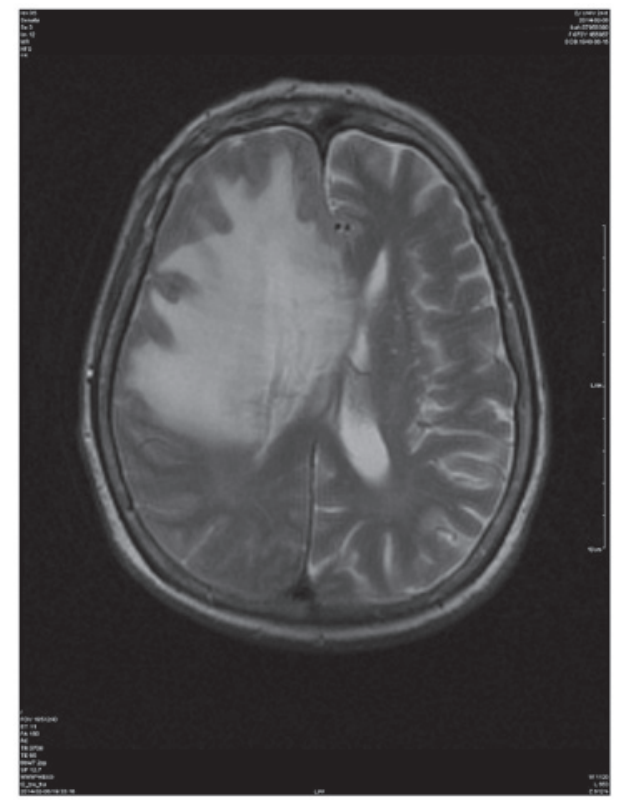

Figure 1. Contrast-enhanced magnetic resonance imaging prior to epidermal growth factor receptor-tyrosine kinase inhibitor treatment, showing brain metastases destroying the frontoparietal bone plate and diploe. The right anterior horn of the lateral ventricle appears compressed and the midline is shifted to the left.

limb and 1/5 in the left lower limb. The right-sided muscle strength was $5 / 5$. The right patellar tendon reflex was rated as $2+$, but was absent on the left side. The Babinski sign was negative. The patient underwent an emergency brain MRI scan, which revealed that the brain metastatic lesion had grown significantly compared with the original scan. Contrast-enhanced MRI showed T1 and T2 hyperintense changes in the superior frontal gyrus, as well as enhancement in the nodular zones of the meninges surrounded with cerebral edema, destruction of the frontoparietal bone plate and diploe. The right anterior horn of the lateral ventricle was compressed and the midline was shifted to the left. Imaging diagnoses included frontal bone malignancy with involvement of the superior frontal gyrus, which was considered as metastasis (Fig. 1).

D-glycerol and steroids were initially administered, but this treatment was ineffective. Moreover, brain surgery was strongly recommended to avoid cerebral herniation; however, the patient's family rejected surgical intervention. Administration of the EGFR-TKI icotinib (125 mg, p.o. 3 times/day) was then recommended, to which the family agreed. Surprisingly, the patient's left body function progressively improved after taking 4 tablets. The physical examination revealed that the muscle strength of the left upper limb was $2 / 5$ and of the left lower limb 3/5. The Babinski sign was negative. After $48 \mathrm{~h}$, the left body dysfunction had resolved. In addition, the dimensions of the horn-like lesion on the patient's forehead markedly decreased, from $18.3 \times 2.3$ to $12.2 \times 1.5 \mathrm{~cm}$. After 4 days of observation, the patient was discharged.

Over 4 months later, the patient underwent a follow-up brain MRI scan, which revealed that the metastatic lesion was significantly smaller compared with prior to treatment (Fig. 2). In October, 2014, the patient remained alive and was continuing icotinib therapy, without other chemotherapies.

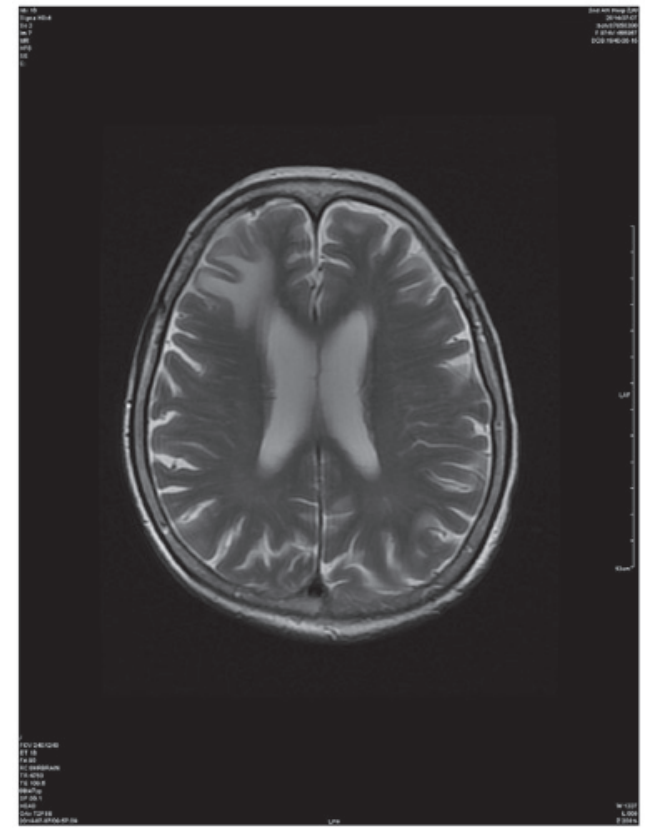

Figure 2. Contrast-enhanced magnetic resonance imaging after 4 months of epidermal growth factor receptor-tyrosine kinase inhibitor treatment, showing substantial regression of the metastatic lesion.

\section{Discussion}

Approximately 20-40\% of NSCLC patients develop BM, which is associated with a poor prognosis. The majority of these patients survive for 3-6 months and the 1-year survival rate is $10 \%(4,8,9)$. Considerable efforts have been made to explore additional or alternative treatment modalities to improve survival, quality of life and local tumor control.

For patients with a single metastatic lesion in the brain, whole-brain radiation therapy (WBRT) is the standard of care, and is often combined with chemotherapy or surgery. When combined with surgical resection, WBRT reduces the risk or relapse and may increase the overall survival of patients with BM $(5,10)$. Moreover, the combined treatment of pemetrexed-cisplatin with concurrent WBRT has been proven effective in patients with NSCLC and BM (11). However, these treatment protocols require the patients to be in relatively good health (i.e., high Karnofsky performance status score) prior to treatment. If a patient is in poor health, as is common with advanced NSCLC, fewer treatment options are available.

In the present case, the patient had a large metastatic lesion in the brain, which invaded the skull in the area of the forehead and caused a midline shift, potentially increasing the risk of increased intracranial pressure (IICP). Glycerol and steroid therapy was not effective in this patient. Given the patient's severe conscious impairment and increasing IICP, she was in an emergency situation requiring a rapid response. Ideally, local control of tumor progression by surgical removal, with or without radiation or radiosurgery, may be the first or optimal method to manage IICP, as illustrated by a previous case of a 57-year-old female patient with a large BM who was succesfully treated with macroscopically complete excision of the brain tumor and postoperative radiotherapy (12). However, in our case, our patient had a poor baseline performance 
status, she was elderly, and the large lung tumor caused left lung atelectasis and invaded the left pulmonary artery, which further increased the risk associated with anesthesia and surgery. Second, edema and brain injury following surgery or radiotherapy is highly possible, potentially compromising the patient's quality of life. The primary tumor in the lung was not sensitive to radiotherapy; therefore surgery and WBRT were not considered to be a safe and effective option for this patient.

As an alternative to WBRT, other therapeutic approaches are available to treat BM. Drugs targeting mutated EGFR and anaplastic lymphoma kinase (ALK) genes have been highly effective when administered systemically $(13,14)$. Several reports demonstrated that gefitinib or icotinib are associated with a favorable response in lung adenocarcinoma with BM, particularly in cases harboring EGFR mutations, such as our patient (15-19). EGFR-TKIs are reported to cross the blood-brain barrier, although it has been suggested that TKI concentration is reduced in the central nervous system compared with the serum levels (20). In such cases, increased doses may improve the efficacy (21). However, in the present case, the standard dose of icotinib quickly relieved the patient's symptoms and suppressed the growth of the metastatic lesion for at least 4 months.

The current standard approach to the management of a life-threatening BM is surgery and radiotherapy. In the present case, we demonstrated that NSCLC with BM may be efficiently treated with the EGFR-TKI icotinib. A standard dose of icotinib reduced the size of the brain lesion and relieved the patient's symptoms as quickly as surgery. Icotinib decreased the size of the protrusion on the patient's forehead as well as the size of the brain mass in $\sim 12 \mathrm{~h}$, which was confirmed by recovery of left body function. This rapid effect may be attributed to two reasons: First, genetic analysis of the primary tumor identified an exon 19 mutation in the EGFR gene. It has been reported that primary tumor EGFR status is a good surrogate for EGFR mutation status of the BM, with a concordance rate of $93.3 \%$ (22). Second, our patient's brain had a large lesion, which disrupted the frontoparietal bone plate and diploe. This damage may have facilitated the transport of icotinib across the blood-brain barrier.

Therefore, our case suggests that EGFR-TKI treatment may be a quick, safe and effective option for patients with BM, particularly those harboring an EGFR mutation, with a poor functional status, or in an emergency situation.

\section{Acknowledgements}

The present study was supported in part by Department of Education of Zhejiang province (grand no. Y201121356).

\section{References}

1. Jemal A, Bray F, Center MM, Ferlay J, Ward E and Forman D: Global cancer statistics. CA Cancer J Clin 61: 69-90, 2011.

2. Schuette W: Treatment of brain metastases from lung cancer: Chemotherapy. Lung Cancer 45 (Suppl 2): S253-S257, 2004.

3. Langer CJ and Mehta MP: Current management of brain metastases, with a focus on systemic options. J Clin Oncol 23: 6207-6219, 2005.
4. Patchell RA, Tibbs PA, Walsh JW, Dempsey RJ, Maruyama Y, Kryscio RJ, Markesbery WR, Macdonald JS and Young B: A randomized trial of surgery in the treatment of single metastases to the brain. N Engl J Med 322: 494-500, 1990.

5. Patchell RA, Tibbs PA, Regine WF, Dempsey RJ, Mohiuddin M, Kryscio RJ, Markesbery WR, Foon KA and Young B: Postoperative radiotherapy in the treatment of single metastases to the brain: A randomized trial. JAMA 280: 1485-1489, 1998.

6. Hazard LJ, Jensen RL and Shrieve DC: Role of stereotactic radiosurgery in the treatment of brain metastases. Am J Clin Oncol 28: 403-410, 2005.

7. Qin H, Pan F, Li J, Zhang X, Liang H and Ruan Z: Whole brain radiotherapy plus concurrent chemotherapy in non-small cell lung cancer patients with brain metastases: A meta-analysis. PLoS One 9: e111475, 2014.

8. Hsiung CY, Leung SW, Wang CJ, Lo SK, Chen HC, Sun LM and Fang FM: The prognostic factors of lung cancer patients with brain metastases treated with radiotherapy. J Neurooncol 36: 71-77, 1998.

9. Lagerwaard FJ, Levendag PC, Nowak PJ, Eijkenboom WM, Hanssens PE and Schmitz PI: Identification of prognostic factors in patients with brain metastases: A review of 1292 patients. Int J Radiat Oncol Biol Phys 43: 795-803, 1999.

10. Armstrong JG, Wronski M, Galicich J, Arbit E, Leibel SA and Burt M: Postoperative radiation for lung cancer metastatic to the brain. J Clin Oncol 12: 2340-2344, 1994.

11. Dinglin XX, Huang Y, Liu H, Zeng YD, Hou X and Chen LK: Pemetrexed and cisplatin combination with concurrent whole brain radiotherapy in patients with brain metastases of lung adenocarcinoma: A single-arm phase II clinical trial. J Neurooncol 112: 461-466, 2013.

12. Kataoka K, Uejima T, Niiyama K, Kuroda R, Arita N, Ioku M, Yamada K, Taneda M and Hayakawa T: Long survival after removal of a huge brain tumor which had metastasized from a lung cancer: Case report. Neurological Surgery 22: 339-341, 1994 (In Japanese).

13. Ettinger DS, Akerley W, Borghaei H, Chang AC, Cheney RT, Chirieac LR, D'Amico TA, Demmy TL, Govindan R, Grannis FW Jr, et al; National Comprehensive Cancer Network: Non-small cell lung cancer, version 2. 2013. J Natl Compr Canc Netw 11: 645-653; quiz 653, 2013.

14. Shaw AT, Kim DW, Nakagawa K, Seto T, Crinó L, Ahn MJ, De Pas T, Besse B, Solomon BJ, Blackhall F, et al: Crizotinib versus chemotherapy in advanced ALK-positive lung cancer. N Engl J Med 368: 2385-2394, 2013.

15. Ceresoli GL, Cappuzzo F, Gregorc V, Bartolini S, Crinò L and Villa E: Gefitinib in patients with brain metastases from non-small-cell lung cancer: A prospective trial. Ann Oncol 15: 1042-1047, 2004.

16. Park SJ, Kim HT, Lee DH, Kim KP, Kim SW, Suh C and Lee JS: Efficacy of epidermal growth factor receptor tyrosine kinase inhibitors for brain metastasis in non-small cell lung cancer patients harboring either exon 19 or 21 mutation. Lung Cancer 77: 556-560, 2012.

17. Jung YH, Han CW, Jung YD, Cho YY and Han DJ: Complete remission of brain metastases in non-small cell lung cancer patients harboring an EGFR mutation treated with tyrosine kinase inhibitor without radiotherapy: A report of 3 cases. Case Rep Oncol 7: 149-154, 2014.

18. Iuchi T, Shingyoji M, Sakaida T, Hatano K, Nagano O, Itakura M, Kageyama H, Yokoi S, Hasegawa Y, Kawasaki K and Iizasa T: Phase II trial of gefitinib alone without radiation therapy for Japanese patients with brain metastases from EGFR-mutant lung adenocarcinoma. Lung Cancer 82: 282-287, 2013.

19. Sun X and Zheng Y: Retreatment with icotinib in a patient with metastatic lung adenocarcinoma. Tumori 99: e124-e126, 2013.

20. Zhao J, Chen M, Zhong W, Zhang L, Li L, Xiao Y, Nie L, Hu P and Wang M: Cerebrospinal fluid concentrations of gefitinib in patients with lung adenocarcinoma. Clin Lung Cancer 14: 188-193, 2013.

21. Yuan Y, Tan C, Li M, Shen H, Fang X, Hu Y and Ma S: Activity of pemetrexed and high-dose gefitinib in an EGFR-mutated lung adenocarcinoma with brain and leptomeningeal metastasis after response to gefitinib. World J Surg Oncol 10: 235, 2012.

22. Luo D, Ye X, Hu Z, Peng K, Song Y, Yin X, Zhu G, Ji Q and Peng Y: EGFR mutation status and its impact on survival of Chinese non-small cell lung cancer patients with brain metastases. Tumour Biol 35: 2437-2444, 2014. 